\title{
Staat, Gesellschaft und grüne Transformationen im globalen Süden
}

\author{
Die Anbahnung und Umsetzung grüner Trans- \\ formationen ist ein komplexer, sektoren- und \\ ebenenübergreifender Prozess, der auf die \\ Unterstützung mächtiger Akteurskonstellationen \\ angewiesen ist. Solche Transformationen müssen \\ von staatlichen Akteuren angestoßen oder \\ begleitet werden, wie das Beispiel der "grünen“ \\ Republik Costa Rica zeigt. \\ Von Linda Wallbott und Markus Lederer
}

Ebene diese Politikversuche zu schnell als irrelevant oder ineffektiv aburteilen, sondern uns vielmehr auf die Potenziale und die Bedingungen einer erfolgreichen Umsetzung von grünen Transformationsprozessen konzentrieren. Im Folgenden werden wir daher zunächst näher auf unsere Terminologie eingehen und dann ein analytisches Gerüst skizzieren, in dem wir das Hauptaugenmerk auf die Frage legen, welche Akteure eine zentrale Rolle übernehmen (sollten) und in welchem Verhältnis staatliche und nichtstaatliche - auch privatwirtschaftliche - Akteure in diesem Prozess zueinander stehen. Diese Aspekte werden durch erste empirische Evidenzen eines in ternationalen und interdisziplinären Forschungsprojekts zum Thema und anhand des Fallbeispiels Costa Rica verdeutlicht [1].

\section{Von Nachhaltigkeit zu Transformation: wenn nicht das, was dann?}

Seit den 1980ern dominiert der Nachhaltigkeitsbegriff die ökologischen und entwicklungspolitischen Debatten in Wissenschaft und Praxis. Die Brundtland-Kommission der Vereinten Nationen definierte nachhaltige Entwicklung in ihrem einschlägigen Bericht „Unsere gemeinsame Zukunft“ (Our common future) im Jahr 1987 als einen intergenerationalen Prozess, durch den die Bedürfnisse der Gegenwart befriedigt werden, ohne dass künftigen Generationen die Chance genommen wird, dies ebenfalls zu erfahren (World Commission on Environment and Development [WCED] 1987). Mit der Konferenz von Rio im Jahre 1992 hatte das Prinzip offiziell Einzug in die internationale Politik gehalten und konnte zumindest vorübergehend den bestehenden Nord-Süd-Konflikt überbrücken. Der Nachhaltigkeitsbegriff dominierte im Folgenden die internationale Umweltpolitik und entwickelte sich auch in der Forschung zu einem wichtigen Paradigma. Ein wesentlicher Bestandteil des Konzepts ist die Annahme, dass Ökologie, Soziales und Ökonomie gleichberechtigte Dimensionen sind und sich gegenseitig bedingen.

In Abgrenzung dazu stehen bei alternativen Konzepten wie "grüner Ökonomie“ oder „grünem Wachstum“ vor allem wirtschaftliche Akteure und Prozesse im Fokus. Die Begriffe wurden, nicht zuletzt durch das persönliche Engagement des vormaligen Exekutivdirektors des UN-Umweltprogramms, Achim Steiner, vor allem von UNEP (UNEP 2011) und der OECD (OECD 2011) geprägt. Auf der Konferenz Rio+20 der Verein- 
ten Nationen im Jahre 2012 erlangte speziell der Green-Economy-Begriff größere Prominenz. Die Konzepte sind jedoch stark umstritten, da nicht nur semantisch die ökonomische Dimension eindeutig im Vordergrund steht (Brand 2012). Gleiches gilt für das verwandte Konzept der kohlenstoffarmen Entwicklung (low carbon development), das unter anderem von der einflussreichen Initiative für eine „New Climate Economy“ als Win-win-Szenario für Wirtschaft und Umwelt propagiert wird. Inwieweit unterscheidet sich nun der Begriff der grünen Transformation von den bisher genannten Konzepten?

Mit diesem neuen Modell wird zum einen an die Nachhaltigkeitsdebatte angeknüpft, insofern als auch hier die Interdependenz der drei Dimensionen Ökologie, Ökonomie und Soziales im Vordergrund steht. In Anlehnung an Polanyis Arbeit zur „Great Transformation“ (Polanyi 1957 [1944]) werden im Unterschied zum klassischen Nachhaltigkeitsbegriff aber tiefgreifende strukturelle gesellschaftliche Veränderungen impliziert. Diese standen bis dato so nicht im Fokus. Es geht bei grünen Transformationen also nicht um singuläre oder ad hoc beziehungsweise in einzelnen Themenfeldern wie zum Beispiel dem Emissionsbereich auftretende Veränderungen der Umwelt- oder Entwicklungspolitik. Vielmehr sollen solche Maßnahmen geplant und umgesetzt werden, die auf den institutionellen Umbau von Gesellschaften abzielen (WBG U 2012). „Transformation“ meint politisch gestalteten Wandel. Dies impliziert sektoren- sowie ebenenübergreifende Interventionen und Veränderungen (Schmitz/Becker 2013; Urban 2014). Sehr anschaulich hat die Ökonomin Kate Raworth vom Environmental Change Institute der Universität Oxford die Verbundenheit des Sozialen, Ökologischen und Ökonomischen in ihrem „Doughnut“-Modell zusammengebracht. Sie betrachtet die Geschehnisse der Welt durch einen Doughnut, dessen äußere Linie durch die auch von Rockström et al. (2009) beschriebenen natürlichen (Ressourcen-)Grenzen des Planeten (planetary boundaries) gekennzeichnet sind. Der innere Ring hingegen ist definiert durch soziale Gerechtigkeit und Menschenrechte. Im Unterschied zum sogenannten Kapitalstockmodell der Nachhaltigkeit, demzufolge all das nachhaltig ist, was vom Ertrag und nicht von den Zinsen lebt, wird hier aber die zyklische Verbundenheit betont und somit ausgeschlossen, dass das Handeln „auf Pump“ in einer Dimension durch erhöhte Erträge in einer anderen Dimension kompensiert werden könnte. Grüne Transformationen, wie wir sie verstehen, sollen also, auch im Licht des Doughnut-Modells betrachtet, stets Veränderungen der betroffenen Politikfelder sowie der verschiedenen Dimensionen von Nachhaltigkeit implizieren. Wir konzipieren dabei des Weiteren grüne Transformationen als Veränderungen in verschiedenen Stadien des Politikprozesses. Die Effektivität politischen Handelns kann demnach anhand dreier Kategorien untersucht werden, nämlich der Agenda-Festlegung und Formulierung von offiziellen Dokumenten (output), der entsprechenden Ergreifung von konkreten Maßnahmen zur Umsetzung (outcome) und des tatsächlichen Einflusses dieser Maßnahmen, zum Beispiel auf das Niveau von Treibhausgasemissionen (impact). Hieraus ergibt sich ein mehrschichtiges Modell grüner Transformationen.

Beispiele früherer und ähnlich radikaler Transformationsprozesse aus anderen Politikbereichen sind die industrielle Revolution, die Abschaffung der Sklaverei oder die Etablierung von Wohlfahrtsstaaten. In all diesen Fällen fand ein tiefgreifender Norm- und Politikwandel statt, welcher weder zeitlich linear noch in allen Gesellschaften nach gleichem Muster ablief. Die Komplexität und vielschichtige Dynamik solcher Entwicklungen sollte sicherlich grundsätzlich anerkannt werden, statt davon auszugehen, dass es nur einen idealen Weg geben könne und es daher keinerlei Politisierung von technologischen Lösungen bedürfe, wie es beispielsweise in der Bundesrepublik Deutschland häufig bei der Diskussion um ökologische Reformprozesse oder die Energiewende scheint. Wir möchten hingegen argumentieren, dass Beobachter/innen und Analyst/innen gleichermaßen einen offenen Blick für die Vielschichtigkeit und Umstrittenheit von Konzeptionen der Probleme und Lösungen von Umweltfragen bewahren sollten. Dort, wo sich die Voraussetzungen unterscheiden, müssen auch verschiedene Pläne her, denn one size fits all gibt es nicht. Mittels dieses Verständnisses wird es möglich, die Bedeutung nationaler Kontextbedingungen und Traditionen für die Gestaltung von Nachhaltigkeitsentwicklungen zu erfassen. Ebenso sind Transformationen, unserem Verständnis nach, immer unabgeschlossen, das heißt, sie sind prinzipiell umkehrbar und ebenso Ergebnis politischen Handelns wie auch stetiger Prozess. Diese analytische Offenheit hat in der politikwissenschaftlichen Debatte beispielsweise ermöglicht, die Bedingungen und Effekte der verschiedenen Varianten von Wohlfahrtsstaatlichkeit zu verstehen, deren Weiterentwicklung nur selten einer ausschließlich funktionalen Logik folgte (Meadowcroft 2005). Man vergleiche nur die skandinavischen Modelle mit den Formen im europäischen Süden. Stattdessen wurden Veränderungen oftmals von harten - teilweise auch gewaltförmigen - Auseinandersetzungen begleitet. Ohne den Druck von Gewerkschaften und der Sozialdemokratie wäre Bismarck sicher nicht auf die Idee verfallen, erste wohlfahrtsstaatliche Maßnahmen einzuführen. Wir wollen an dieser Stelle keine vollständige Analogie über die Entwicklungspfade grüner Transformationen in empirischer oder gar normativer Lesart geben. Gleichwohl ist eine Spezifikation des Transformationsbegriffs hilfreich, um auch die politisch relevante Tiefe und Reichweite der angedachten Veränderungen zu betonen.

\section{Staaten, Gesellschaften und Unternehmen in globaler Perspektive: Wer macht was?}

Transformationen brauchen neben revolutionären Ideen oder neuen Techniken aber eben auch Akteure, die Veränderungen politisch vorantreiben, gestalten und umsetzen. Während die klassische Nachhaltigkeitsdebatte meist zivilgesellschaftliche beziehungsweise partnerschaftliche Akteurskonstellationen in den Fokus nahm und die Diskussion zur grünen 
Ökonomie vor allem auf die Beiträge und Initiativen privatwirtschaftlicher Akteure setzt, so gibt es in der aktuellen Literatur zu grünen Transformationen eine Rückbesinnung und (erneute) Hinwendung auf den Staat - und hier insbesondere Ministerien und nachgelagerte Verwaltungen (Death 2015). Auch für Unternehmen tragen Regularien schließlich zur Erwartungs- und Investitionssicherheit bei. Es gibt daher eine lebendige Debatte um „grüne Staaten“ (green states) (Eckersley 2004), wobei diese primär auf den globalen Norden fokussiert und entsprechende Studien, vor allem in vergleichender Perspektive, für den Süden eher rar gesät sind. Dies überrascht insofern, als viele Staaten des globalen Südens eben keine Zeichen „zerfallender Staatlichkeit" aufweisen, wie bei oberflächlicher Betrachtung eventuell angenommen werden könnte, sondern die staatlichen Einheiten oftmals ebenfalls prinzipiell über starken Einfluss und Durchgriff auf ihre Gesellschaften verfügen. Dies wurde zum Beispiel bei der ökonomischen Transformation in Ost- und Südostasien deutlich. Die Regierungen dieser im späteren Verlauf als „Tigerstaaten“ bezeichneten vermeintlichen Entwicklungsstaaten wie Südkorea und Taiwan haben im ökonomischen Bereich tiefgreifenden Wandel in ihren Gesellschaftsstrukturen initiiert und damit lang anhaltendes Wachstum anschieben können (Johnson 1982; Wade 2004).

Unter welchen Bedingungen aber gelingen solche staatszentrierten Transformationsprozesse? Die Literatur hat vier essentielle Elemente herausgearbeitet, die hier von Bedeutung sind. Erstens braucht es eine Elite, die in der Regierung des betreffenden Staates ein Instrument sieht, das gleichwohl nicht nur zur Mehrung des eigenen privaten Nutzens eingesetzt wird (Evans 1995). Dies impliziert einen politischen Minimalkonsens darüber, dass es zumindest eine partielle Gemeinwohlorientierung der herrschenden Klasse gibt und Wandel in der Art zu gestalten sei, dass langfristig nicht nur wenige Einzelne profitieren.

Zweitens ist neben einer solchen „staatstragenden Klasse“ auch ein effektiver und mit Kapazitäten ausgestatteter Verwaltungsstab notwendig. So haben verschiedene neo-institutionelle Autor/innen mit Bezug auf Weber die Bedeutung von Bürokratien für den Erfolg ökonomischer Entwicklung betont, da privatwirtschaftliche Akteure weder die für Wachstumsprozesse notwendigen öffentlichen Güter bereitstellen würden, noch negative Externalitäten beheben könnten. So argumentiert etwa Fukuyama recht plakativ: „[B]efore governments can be constrained, they have to generate power to actually do things. States, in other words, have to be able to govern " (Fukuyama 2014, S. 52). Zwar wurden sicherlich nicht alle politischen Transformationen durch einen Weberschen Anstaltsstaat initiiert, aber jegliche langfristige politische Herrschaft braucht eine Verwaltung („Herrschaft ist im Alltag: Verwaltung“ [Max Weber], Fukuyama 2014). Wir würden an dieser Stelle argumentieren, dass tatsächliche Transformation im Sinne tiefgreifenden Wandels eben auf verschiedenen institutionellen und sozial-politisch-ökonomischen Säulen ruhen muss und daher schlussendlich auch auf (unterstützende) Herrschaft angewie- sen ist. Evans hat am Beispiel der ökonomischen Wachstumserfolge in Südostasien gezeigt, dass administrative Akteure als Geburtshelfer weitreichenden Wandels auch jenseits ihrer eigenen vier Bürowände Einfluss haben (Evans 1995). Voraussetzung ist allerdings, dass sie mit den entsprechenden (i)materiellen Kapazitäten und Befugnissen, etwa einem offiziellen Mandat als Teil der institutionellen Rahmenbedingungen, ausgestattet sind.

Drittens sind Verwaltungen immer in gesellschaftliche Kontexte und Machtspiele eingebettet, sodass sich die Frage stellt, über wie viel Autonomie sie tatsächlich verfügen (beispielsweise, um Ideen und Strategien zu grünen Transformationsprozessen zu entwickeln und umzusetzen). In diesem Zusammenhang bezeichnet der Begriff der eingebetteten Autonomie (embedded autonomy) eine Situation, in der Bürokratien auf der einen Seite in der Lage sind, sich eine gewisse Unabhängigkeit von zivilgesellschaftlichem Druck zu bewahren; sie aber auf der anderen Seite nah genug an den relevanten politischen Entscheidungsträgern dran sind, um gegebenenfalls auch Partikularinteressen zur Durchsetzung zu verhelfen (ibid.). Wichtig ist zu betonen, dass an dieser Stelle kein normatives Argument für von gesellschaftlichen Prozessen weitgehend losgelöste Bürokratien eingebracht werden soll (Evans 2008). Allerdings ist zu konstatieren, dass gerade im Angesicht der ökologischen Krise der Ruf nach autokratischen Herrschaftselementen immer wieder auftaucht (für einen Überblick über die Literatur, siehe Beeson 2010), sodass sich in analytischer Hinsicht die Frage nach den genauen Konstellationen von Institutionen und Akteuren stellt, die in möglichst effektiver, aber eben auch legitimer Art grüne Transformationsprozesse anstoßen und implementieren können.

Schließlich ist, viertens, neben den bereits genannten Elementen der Kapazität und der Autonomie auch wichtig, dass Verwaltungen in Prozesse der Gewaltenteilung eingebunden sind (Fukuyama 2014, S. 52). Das verwandte Konzept der Rechtssicherheit kann hier einerseits runtergebrochen werden auf die Berechenbarkeit institutioneller Verfahren; aber auch allgemeine Transparenzvorgaben spielen eine Rolle. So müssen inklusive politische Institutionen einerseits stark genug sein, um politische Prozesse zu steuern; sie sollen aber gleichzeitig auch kontrollierbar bleiben, um Rentierverhalten und das ausschließliche Verfolgen partikularer Interessen zu vermeiden (Acemoglu/Robinson 2012). Die Idee grüner Transformationen greift somit klassische politikwissenschaftliche Konzepte auf und wendet sie auf aktuelle und drängende gesamtgesellschaftliche Fragen an.

\section{Grüne Transformationen in Costa Rica}

Im Folgenden möchten wir nun am Beispiel von Costa Rica und an ausgewählten Sektoren exemplarisch erläutern, wie grüne Transformationen angelegt und potenziell ausgestaltet sein können. Dabei gehen wir auch auf die Frage ein, wie die bisher vollzogenen Schritte zu bewerten sind und welche Rolle 
die oben skizzierten Einflüsse staatlicher Akteure und ihrer Interaktion mit gesellschaftlichen Gruppen bei den nationalen Entwicklungen gespielt haben.

Warum nun aber dieses Land? Wir konzentrieren uns in diesem Beitrag auf Costa Rica, da es sowohl in regionaler als auch in globaler Perspektive als Vorreiter der Formulierung grüner Politiken betrachtet werden kann. Dies machen wir an dieser Stelle nicht nur an der Dichte, sondern auch an der Reichweite der entsprechenden Dokumente fest.

So hatte Costa Rica im Jahr 2007 unter Präsident Oscar Arias Sánchez angekündigt, bis 2021 „kohlenstoff-neutral“ zu sein, das heißt, entweder keine neuen Treibhausgasemissionen zu produzieren (im Vergleich zum Basisjahr 2005) oder sie vollständig zu kompensieren. Dieses Ziel wurde 2009 in der Nationalen Klimawandel-Strategie formalisiert. Im September 2015 wurde beim Klimagipfel der Vereinten Nationen in Paris außerdem das Ziel ausgegeben, bis 2030 die absoluten Kohlendioxid-Emissionen um $25 \%$ zu reduzieren (Referenzjahr 2012) (Araya 2015). Damit ist Costa Rica eines von lediglich fünf Ländern, die im Übrigen mit Marokko, Bhutan, Gambia und Äthiopien alle aus der Gruppe der nichtindustrialisierten Länder stammen, deren national festgelegte Beiträge zum Klimaschutz (Nationally Determined Contributions, NDCs) von Beobachtern des Prozesses als ausreichend (sufficient) eingestuft wurden (Climate Action Tracker 2015). In Bezug auf output steht Costa Rica also relativ gut da. Wie aber ist es um die anderen beiden Stufen von Effektivität, outcome und impact, bestellt? Zur Beantwortung dieser Frage blicken wir auf Entwicklungen in den Politikfeldern Energie und Landnutzung.

Insgesamt ist es dem Land nach Angaben des nationalen Energieversorgers ICE (Instituto Costarricense de Electricidad) gelungen, im Jahr 2015 seinen Bedarf an Elektrizität für mehr als 250 Tage zu $100 \%$ aus erneuerbaren Energiequellen zu decken (Dyer 2015). Dies wurde durch die überdurchschnittlich starken Regenfälle im ersten Quartal und die dadurch gut gefüllten Staudämme des Landes ermöglicht. Im Vorjahr allerdings litt das Land unter einer extremen Trockenperiode und es ergab sich die paradoxe Situation, dass Costa Rica erst durch extreme Wetterschwankungen in die Lage versetzt wurde, fast ausschließlich Ökostrom zu produzieren (2016 waren es schließlich sogar 271 Tage, an denen die Stromerzeugung durch Wind- und Wasserkraft sowie Geothermie bestritten werden konnte, und lag die Quote insgesamt bei $98 \%$; Fendt 2017). Nicht fossilen Energiequellen kommt bei der Umsetzung des Klimaneutralitäts-Vorhabens einerseits eine gestiegene Bedeutung zu. Andererseits muss konstatiert werden, dass Anlagen zur Erzeugung von Wasserkraft (Staudämme; in Costa Rica für $70 \%$ der Stromerzeugung verantwortlich) oder nachwachsende Brennstoffe (Biomasse) in der Regel nicht ohne negative Wechselwirkungen im sozialen oder ökologischen Bereich auskommen.

Zudem ist es nach wie vor so, dass Costa Rica etwa 70\% der national genutzten fossilen Brennstoffe für den Transport aufwendet (Fendt 2015) und der relative Anteil des Transportsek-

\section{„Die Idee grüner Transforma- tionen greift klassische politikwissenschaftliche Konzepte auf und wendet sie auf aktuelle und drängende gesamt- gesellschaftliche Fragen an. “}

tors an den nationalen Treibhausgasemissionen ist beachtlich: Er stieg von 1,69\% in 1990 auf 68,34\% in 2015 an (UNFCCC, o. J.). Gleichzeitig hat sich die absolute Energie-Konsummenge des Transportsektors im selben Zeitraum mehr als verdoppelt und bis ins Jahr 2014 sogar verdreifacht (IEA o. J.). Darüber hinaus wächst der Privatbesitz von Autos pro Jahr um circa 5\% stetig weiter an und das Land wendet ca. $4 \%$ seines Bruttosozialprodukts für den Import von Öl auf (Utgård/Araya 2016). In der Konsequenz bedeutet das: Will Costa Rica tatsächlich den Weg einer grünen Transformation beschreiten und die ausgegebenen Politiken glaubhaft umsetzen, muss das Land von seinem Entwicklungsweg der letzten 25 Jahre abweichen und sein Transportwesen revolutionieren. Das würde Investitionen in die öffentliche Infrastruktur und in Fahrzeuge des öffentlichen Verkehrssystems sowie Förderung und Schaffung der politischen, marktwirtschaftlichen und infrastrukturellen Voraussetzungen für private und öffentliche Elektromobilität erforderlich machen.

Neben dem Transportsektor ist der Bereich Landnutzung, vor allem Forst- und Landwirtschaft, mit über 30\% hauptverantwortlich für den Ausstoß von Treibhausgasen in Costa Rica. In beiden Bereichen gibt es "grüne“ Entwicklungen, wobei Costa Rica insbesondere durch seine Waldpolitik international bekannt wurde. So initiierte das Land 2005 gemeinsam mit Papua-Neuguinea einen internationalen Verhandlungsprozess im Kontext der Klimarahmenkonvention der Vereinten Nationen, der wenig später, in 2010, zur Verabschiedung eines Instruments gegen Entwaldung und für Wiederaufforstung (Reducing Emissions from Deforestation and Forest Degradation, REDD+) führen sollte (Turnhout et al. 2016). Dieses Engagement hatte jedoch auch nationale Entsprechungen. So gelang es, die Waldfläche in Costa Rica im Vergleich zu Anfang der 1980er Jahre zu verdoppeln und $25 \%$ des Landes als Nationalpark oder geschützte Fläche zu deklarieren. Von internationalen Gebern, der nationalen Regierung und großen Teilen der Zivilgesellschaft wurde die Einführung von Standards für Nachhaltiges Waldmanagement (Sustainable Forest Management) Mitte der 1980er und eines Kompensationssystems für 
Ökosystem-Dienstleistungen (Payment for Ecosystem Services, kurz PES) in den 1990er Jahren unterstützt. Mittlerweile importiert Costa Rica jedoch mehr Holz als es exportiert: 2015 betrug das Verhältnis 77 Millionen US\$ zu 102 Millionen US\$ (Milla 2017). Dies weist darauf hin, dass bei der Analyse grüner Transformationsprozesse auch regionale Dynamiken und (wirtschaftliche) Beziehungen berücksichtigt werden sollten. Denn wenn es sich lediglich um eine geografische oder zeitliche Verschiebung von Externalitäten (zum Beispiel aufgrund stabilen Konsumverhaltens) handelt, kann von nachhaltigem Wandel kaum gesprochen werden.

Dabei geht der Blick nach vorne, denn 2016 wurden zwei weitere wichtige Dokumente verabschiedet (output), deren Folgewirkungen (outcome und impact) zum jetzigen Zeitpunkt gleichwohl noch nicht abschließend beurteilt werden können: zunächst verabschiedete die Interministerielle Kommission des Ministeriums für Landwirtschaft (Ministeria de Agricultura $y$ Ganaderia, MAG) und des Ministeriums für Umwelt und Energie (Ministerio de Ambiente y Energía, MINAE), das auch für die nationale Klimastrategie verantwortlich zeichnet, die Agenda für Landwirtschaft und Umwelt (Agenda Agro-Ambiental). Dann erließ die Regierung die Nationale Strategie zur Rehabilitierung von Produktionsflächen (Estrategia Nacional de Rehabilitación de Paisajes Productivos de Costa Rica). Letztere soll als integrativer Rahmen für die Koordination der Landnutzung zwischen landwirtschaftlichen und forstwirtschaftlichen Sektoren dienen. Gebiete mit hoher Produktivität sollen derart umgestaltet werden, dass Rivalität hinsichtlich potenzieller unternehmerischer Gewinne durch verschiedene Nutzungsarten ausgeschlossen wird. Stattdessen sollen Farmer/innen und Waldbesitzer/innen, unterstützt durch neuartige Zertifizierungssysteme, bisher nicht zugängliche Märkte nutzen beziehungsweise sie erschließen und hierdurch ihre Produktion so umstellen, dass beispielsweise in den Bereichen Kaffee (in 2005 10\% der nationalen Treibhaugasemissionen beziehungsweise ein Anteil von $25 \%$ an allen landwirtschaftlichen Emissionen und Beschäftigung von $8 \%$ aller Arbeitskräfte; G IZ 2015) und Viehhaltung Emissionen eingespart werden können. Weitere wichtige Politikinstrumente sind in diesem Zusammenhang auch die Diffusion innovativer Technologien und Training der entsprechenden Zielgruppen.

\section{Die Rolle des Staates und seine Interaktion mit nichtstaatlichen Akteuren}

Welche Rolle spielten nun aber nationale Eliten, die Kapazität der Verwaltung Costa Ricas, ihre Einbindung in gesellschaftliche Prozesse bei gleichzeitiger relativer Autonomie sowie Gewaltenteilung für die beschriebenen Entwicklungen? Viele Ideen zu einer grünen Transformation wurden von der Zivilgesellschaft oder von internationalen Gebern lanciert. So führte die Weltbank bereits in den 1980ern die ersten debt-fornature-swaps ein, um der Regierung die Schuldenbelastung zu erleichtern und gleichzeitig Schutzgebiete zu fördern. Die poli- tische Führung des Landes trug diese „grüne“ Agenda jedoch von Anfang an mit. Die präsidentielle und öffentlichkeitswirksame Ankündigung sind Teil der jüngeren „grünen“ Geschichte des Landes und die Regierung ist entschlossen, das - auch hinsichtlich des internationalen Tourismus (Campbell 2002) gewinnbringende - Image eines ökologischen Eldorados aufrechtzuerhalten. So wurde Costa Rica bereits Ende der 1990er Jahre und trotz aller Widersprüche und Schwierigkeiten als „grüne Republik“ (Green Republic) bezeichnet (Evans 1999).

Bei der Einschätzung der Leistungsfähigkeit der nationalen Bürokratie wie auch der Offenheit der formalen staatlichen Institutionen für zivilgesellschaftliche Akteure hilft der Transformations-Index der Bertelsmann Stiftung. Für das eher kleine, aber doch recht zentralistisch organisierte Costa Rica, in der Literatur auch schon als „social democratic developmental state“ (Sandbrook et al. 2007) beschrieben, zeigt der Index, dass das Land hinsichtlich der Stabilität seiner Staatlichkeit und staatlichen Handlungsfähigkeit gut aufgestellt ist. Das Beispiel der oben genannten Interministeriellen Kommission weist darauf hin, dass die Administration eine wichtige Rolle bei der Weiterentwicklung von nachhaltigen Prozessen spielt.

Dies gilt ebenso für den Faktor politische Beteiligung, wobei diese Einschätzung durch den Freedom House Index gestützt wird, der die zivilen und politischen Freiheiten in einem Land misst und Costa Rica als „frei“ einstuft. Generell wird häufig argumentiert, dass die Entwicklung grüner Politik durch einen starken Konsens zwischen Politik, Zivilgesellschaft, Wissenschaft und Wirtschaft gestützt wird und konsensorientiert ist (Miranda et al. 2002, S. 73). Vor allem das enge, häufig sehr persönliche aber auch beratungsintensive Verhältnis von politischen Entscheidungsträger/innen und Wissenschaftler/ innen ist auffällig.

Dass es dennoch lohnt, hinter die Fassade erster Eindrücke und aggregierter Indizes zu schauen, wird allerdings deutlich, wenn man sich beispielsweise mit der Situation indigener Gruppen in Costa Rica beschäftigt. So wird Costa Rica seit vielen Jahren für Verletzungen, insbesondere der eigentlich formal anerkannten Territorialrechte der indigenen Gruppen kritisiert. Zwar wurde bereits 1977 das „Gesetz zum Schutz Indigener Völker“ (Ley Indígena) mit dem Ziel verabschiedet, den zunehmenden Landverlust der indigenen Gruppen durch die Verleihung kollektiver Landrechte zu stoppen. Allerdings hapert es an der Umsetzung und Landkonflikte verlaufen auch oftmals gewaltförmig (Ortiz 2014). Die Vereinten Nationen gehen davon aus, dass mindestens $40 \%$ der indigenen Territorien zu 80-98\% illegal besetzt sind und konstatieren „the pattern of pervasive, long-standing and inter-connected violations or denials of the rights of indigenous peoples in the Republic of Costa Rica [...] and the ongoing situation of impunity in which they occur and persist" (OHCHR 2015, S. 1). So gab es denn auch bei der Einführung des nationalen PES-Systems nur wenige effektive Konsultationen mit indigenen Gruppen, obwohl ihre Gebiete sich über rund $6 \%$ des gesamten Staatsgebiets erstrecken. 


\section{Ausblick}

Die skizzierten Beispiele deuten bereits an, dass die Einschätzung und Beurteilung von Wechselwirkungen zwischen den verschiedenen Dimensionen von Nachhaltigkeit, insbesondere hinsichtlich outcome und impact und der Perspektive der Betroffenen, doch Gegenstand differenzierter empirischer Untersuchungen sein sollten.

Weitere Forschung sollte sich vor diesem Hintergrund in komparativer Weise mit grünen Transformationsprozessen in verschiedenen politischen Systemen, der Bedeutung unterschiedlicher Formen von Widerstand und Politisierung sowie interdisziplinär auch stärker mit ihren normativen Dimensionen, also der Frage nach Gerechtigkeitsimplikationen wie der Verteilung von Kosten und Nutzen und fairen Verfahren, beschäftigen.

\section{Anmerkung}

[1] Dieser Artikel entstand im Rahmen des Forschungsprojekts „Green Transformations in the global South (GreeTS): opening the black-box of a pro-active state and the management of sustainability trade-offs in Costa Rica and Vietnam" (www.greets-project.org), das durch die Förderlinie „Europe and Global Challenges“ von der VW Stiftung, Riksbankens Jubileumsfond und Wellcome Trust finanziert wird.

\section{Literatur}

Acemoglu, D./Robinson, J.A. (2012): Why Nations Fail. The Origins of Power, Prosperity, and Poverty. New York, Crown Business.

Araya, M. (2015): First reaction to Costa Rica's draft climate contribution for Paris. www.nivela.org/blog/first-reaction-to-costa-rica-s-draft-climatecontribution-for-paris-2/en. Abgerufen am 31. März 2017.

Beeson, M. (2010): The Coming of Environmental Authoritarianism. In: Environmental Politics 19/2: 276-294.

Brand, U. (2012): Schöne Grüne Welt. Über die Mythen der Green Economy. luxemburg argumente. Rosa Luxemburg Stiftung. Berlin.

Campbell, L. M. (2002): Conservation Narratives in Costa Rica: Conflict and Co-existence. In: Development and Change 33/1: 29-56.

Climate Action Tracker (2015): Costa Rica. www.climateactiontracker.org/ countries/costarica.html. Abgerufen am 31. März 2017.

Death, C. (2015): Four discourses of the green economy in the global South. In: Third World Quarterly 36/12: 2207-2224.

Dyer, Z. (2015): Costa Rica backs away from 2021 carbon neutrality goal. In: The Tico Times. November 30, 2015.

Eckersley, R. (2004): The Green State: Rethinking Democracy and Sovereignty. London, MIT Press.

Evans, P. B. (1995): Embedded Autonomy: State and Industrial Transformation. Princeton, Princeton University Press.

Evans, P. B. (2008): In Search of the 21st Century Developmental State. CGPE Working Paper No. 4. Brighton.

Evans, S. (1999): The green republic: A conservation history of Costa Rica. Austin, University of Texas Press.

Fendt, L. (2015): The truth behind Costa Rica's renewable energy. The Guardian.

Fendt, L. (2017): All that glitters is not green: Costa Rica's renewables conceal dependence on oil. The Guardian.

Fukuyama, F. (2014): Political Order and Political Decay: From the Industrial Revolution to the Globalization of Democracy. London, Farrar, Straus and Giroux.

GIZ (2015): NAMA Coffee Costa Rica. A Tool for Low-Carbon Development. www.giz.de/de/downloads/giz2016_en_Factsheet_NAMA_Cafe.pdf. Abgerufen am 3. April 2017.

Gupta, J. (2007): Globalization, Environmental Challenges and North-South Issues. In: V. Thai, K./Rahm, D./Coggburn, J. D. (Hrsg.): Handbook of Globalization and the Environment. Boca Raton. 449-472.

IEA (o.J.): Costa Rica Balances for 2014. www.iea.org/statistics/ statisticssearch/report/?country=COSTARICA\&product=balances \&year=Select. Abgerufen am 31. März 2017.

Johnson, C. (1982): MITI and the Japanese Miracle: The Growth of Industry Policy 1925-1975. Stanford, Stanford University Press.

Meadowcroft, J. (2005): From Welfare State to Ecostate. In: Barry, J./ Eckersley, R. (Hrsg.): The State and the Global Ecological Crisis. Cambridge, MIT Press. 3-24.

Milla, V. (2017): Green Transformations: Developing a Bio-Economy in Costa Rica. Vortrag CATIE. Turrialba/Costa Rica. 29. März 2017.

Miranda, M./Dieperink, C./Glasbergen, P. (2002): The Social Meaning of Carbon Dioxide Emission Trading Institutional Capacity Building for a Green Market in Costa Rica. In: Environment, Development and Sustainability 4/1: 69-86.

OECD (2011): Towards Green Growth, OECD.

OHCHR (2015): Report on the Grave and Persistent Violation of Indigenous Peoples' Rights in Costa Rica. Genf.

Ortiz, D. A. (2014): Indigenous Leaders in Costa Rica Tell Ban Ki-moon Their Problems. InterPress Service.

Polanyi, Karl (1957 [1944]): The Great Transformation. The political and economic origins of our time. Boston, Beacon Press.

Roberts, T. J./Parks, B. (2007): A Climate of Injustice. Global Inequality, NorthSouth Politics, and Climate Policy. Cambridge, MIT Press.

Rockström, J./Steffen, W., et al. (2009): A safe operating space for humanity. In: Nature 461/7263: 472-475.

Sandbrook, R./Edelman, M. et al. (2007): Social Democracy in the Global Periphery. Cambridge, Cambridge University Press.

Schmitz, H. P./Becker, B. (2013): From Sustainable Development to the Green Transformation - A Rough Guide. Sussex.

Turnhout, E./Gupta, A. et al. (2016): Envisioning REDD+ in a post-Paris era: between evolving expectations and current practice. In: Wiley Interdisciplinary Reviews: Climate Change. 8/1.

UNEP (2011): Towards a Green Economy: Pathways to Sustainable Development and Poverty Eradication. Nairobi, UNEP.

UNFCCC (o. ..): Emissions Summary of Costa Rica. www.unfccc.int/files/ ghg_data/ghg_data_unfccc/ghg_profiles/application/pdf/cri_ghg_profile. pdf. Abgerufen am 31. März 2017.

Urban, F. (2014): Low Carbon Transitions for Developing Countries. Oxon, Earthscan.

Utgård, B./Araya, M. (2016): Pura Vida! Driving Smart Electric Mobility in Costa Rica. www.elbil.no/pura-vida-driving-smart-electric-mobility-incosta-rica. Abgerufen am 31. März 2017.

Wade, R. H. (2004): Governing the Market: Economic Theory and the Role of Government in East Asian Industrialization. Princeton, Princeton University Press.

WBGU (2012): Welt im Wandel - Gesellschaftsvertrag für eine Große Transformation. Berlin, WBGU.

WCED (World Commission on Environment and Development) (1987): Our Common Future: The Brundtland Report. Oxford.

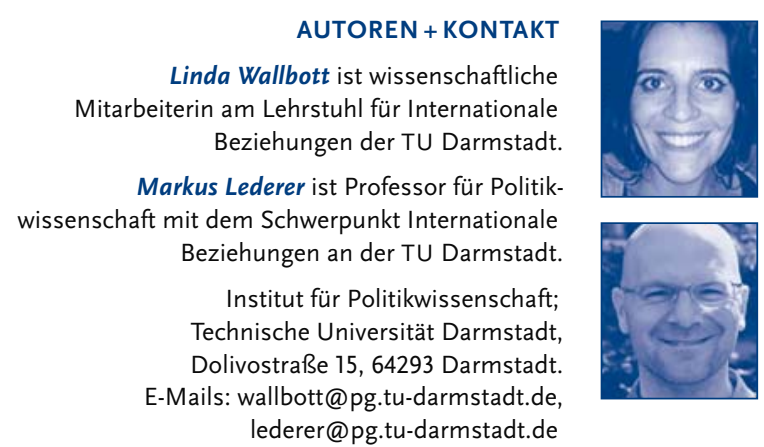

ÖkologischesWirtschaften 2.2017 (32) 